\title{
Conversion from daily to alternate daily corticosteroids in rheumatoid arthritis
}

\author{
M. A. FITZCHARLES, J. HALSEY, AND H. L. F. CURREY \\ From the Department of Rheumatology, the London Hospital, Whitechapel, London E1 IBB
}

SUMMARY An attempt was made to convert 24 patients on corticosteroid treatment from a daily to an alternate daily regimen. Ten patients were successfully converted, 11 failed to convert, and 3 had to be withdrawn for irrelevant reasons. A simple tetracosactrin stimulation test gave some indication of which patients were more likely to convert successfully. Success was not influenced by severity or duration of disease, nor by dose or duration of steroid therapy. Conversion did not influence various clinical and laboratory measures of undesirable steroid side effects, but the follow-up period was probably too short to judge this. The evidence of others suggests that conversion is worth attempting.

There is evidence that in rheumatoid arthritis (RA), alternate daily adrenal corticosteroid (steroid) dosage provides a more favourable therapeutic-totoxic ratio than does the same total dosage given as a daily regimen. There is agreement that, in contrast to daily dosage, alternate daily steroids tend not to suppress hypothalamo-pituitary-adrenal (HPA) axis responsiveness, ${ }^{1}$ and other adverse effects appear to be lessened by alternate daily administration. ${ }^{2-4}$

The popularity of steroids for treating the joint manifestations of RA is decreasing. Nevertheless it seems likely that some patients will continue to be started on this form of therapy. For these there is a good case for starting with alternate daily dosage. For those many rheumatoid patients already taking daily steroids-and unable to manage without them-conversion to an alternate daily regimen is difficult. ${ }^{2}$ An abrupt change may precipitate either an acute exacerbation of joint pains or a steroid withdrawal syndrome. For such patients Fauci has devised a protocol designed to facilitate conversion from a daily to an alternate daily regimen. ${ }^{5}$

Using the protocol we have attempted to convert all suitable patients in our clinic taking at least $5 \mathrm{mg}$ prednisolone daily for control of joint symptoms. The trial was designed to discover what proportion of patients can convert, whether conversion to the same total dose given on alternate days provides

Accepted for publication 26 January 1981

Correspondence to Professor H. L. F. Currey, London Hospital Medical College, Bone and Joint Research Unit, Arthritis and Rheumatism Council Building, 25-29 Ashfield Street, London E1 2AD. comparable control of disease activity, whether there are factors (including a simple adrenal function test) that identify which patients are more likely to be able to convert, and whether conversion actually influences undesirable steroid side effects.

\section{Patients and methods}

All suitable patients attending our rheumatology outpatient clinic and taking at least $5 \mathrm{mg}$ prednisolone daily for classical RA were entered. All were seen regularly by M.A.F. throughout the 24 weeks of the study. In each case it was established that the daily prednisolone dosage had been 'titrated down' to the minimum acceptable. As a simple test of adrenal responsiveness, each patient had plasma cortisol levels determined before and 1 hour after an intravenous injection of $0 \cdot 25 \mathrm{mg}$ tetracosactrin. ${ }^{6}$ Clinical and laboratory measures of disease activity and drug consumption were recorded at each visit, as well as evidence of steroid side effects (weight, blood pressure, blood sugar, bruising, infections).

Conversion was attempted by doubling the 'on' day dose, preferably as a single morning dose. The dose on the ultimate 'off' day was then gradually tapered and finally-if possible-discontinued. The time taken for this was determined by the individual patient's needs. Any increase in joint discomfort was treated by adding further analgesics to the patient's existing regimen of nonsteroidal antiinflammatory drugs (NSAID). Failure was defined as clinical deterioration which persisted for longer than 12 weeks. 


\section{Results}

A total of 24 patients were entered. Their characteristics are shown in Table 1.

Ten of the 24 patients successfully converted to alternate daily steroids over a mean period of 12 weeks. Eleven failed to convert and returned to their previous daily dosage. Three were withdrawn from the study: 2 moved from our area; 1 defaulted before conversion was attempted.

During the first few weeks of attempted conversion all patients experienced increasing stiffness, most prominent on the 'off' or reducing day. In those who failed to convert this stiffness lasted for progressively longer periods, and despite added supplementary medication this became a problem also on the 'on' day.

Those who successfully converted to alternate daily steroids were finally stabilised on the same total dose of prednisolone. By clinical and laboratory criteria their disease activity was the same as when they had been on daily steroids, and their analgesic and NSAID regimen was approximately the same.

There was no correlation between the ability to convert and the dose or duration of steroid treatment, nor with the severity or duration of disease (Table 2). However, of those patients investigated by the tetracosactrin stimulation test all 9 'converters' gave a normal response, while 6 of 10 who failed to respond gave abnormal (inadequate) responses (Table 3).

During the 24 weeks of the study no significant change occurred in the various clinical and laboratory features which were followed as measures of undesirable steroid 'side effects'.

Table 1 Patient characteristics (mean values and range)

\begin{tabular}{lc}
\hline Age (years) & $60 \cdot 5(44-76)$ \\
Duration RA (years) & $17 \cdot 0(7-40)$ \\
Duration steroids (years) & $8 \cdot 5(0 \cdot 2-17)$ \\
Prednisolone dosage (mg) & $6 \cdot 7(5-10)$ \\
Ritchie articular index & $8 \cdot 1(0-24)$ \\
ESR (mm/h) & $44(13-89)$ \\
\hline
\end{tabular}

Twenty-four patients (female 18 ; latex test $\geqslant 1 / 80: 23$ ).

Table 2 Comparison of clinical and laboratory features among patients who converted or failed to convert (mean values)

\begin{tabular}{lcc}
\hline & Converted & $\begin{array}{l}\text { Failed } \\
\text { conversion }\end{array}$ \\
\hline Number & 10 & 11 \\
Age (years) & $61 \cdot 5$ & $60 \cdot 0$ \\
Disease duration (years) & $18 \cdot 8$ & $15 \cdot 2$ \\
Steroid duration (years) & $8 \cdot 0$ & $6 \cdot 0$ \\
Prednisolone dosage (mg) & $6 \cdot 2$ & $7 \cdot 2$ \\
Articular index (Ritchie) & $8 \cdot 9$ & $9 \cdot 3$ \\
ESR (mm/h) & 46 & 42 \\
Morning stiffness (min) & 48 & 49 \\
\hline
\end{tabular}

Table 3 Comparison of adrenal responsiveness between those who achieved conversion and those who failed to convert. A normal response was taken as an increase over baseline serum cortisol levels of at least 200 $\mathrm{nmol} / \mathrm{l}(7 \mu \mathrm{g} / 100 \mathrm{ml}) 1$ hour after administration of $0.25 \mathrm{mg}$ tetracosactrin ${ }^{6}$

\begin{tabular}{lll}
\hline & \multicolumn{2}{l}{$\begin{array}{l}\text { Serum cortisol response to } \\
\text { tetracosactrin }\end{array}$} \\
\cline { 2 - 3 } & Normal & Abnormal \\
\hline Converters $(\mathrm{n}=9)$ & 9 & 0 \\
Failed conversion $(\mathrm{n}=10)$ & 4 & 6 \\
\hline
\end{tabular}

Fisher's exact test $\mathbf{p}<0.05$.

\section{Discussion}

This study was carried out in a clinic in which steroids are used in RA only as a last resort and in which the policy is to titrate the dose down to the minimum acceptable - against the background of full NSAID treatment. Our results suggest that in these circumstances something like $50 \%$ of patients can convert to alternate daily steroids. Once converted, their disease was as well controlled as it had been on the daily regimen. The 'off' day stiffness noted in juvenile chronic arthritis ${ }^{7}$ occurred during conversion, but in those who successfully converted this could be controlled by additional analgesics and NSAID and ceased to be a problem once the alternate daily regimen was established.

Unexpectedly the ability to convert appeared not to be influenced by disease duration or severity, nor by steroid dosage or duration (within the range met here). We did not adequately test hypothalamopituitary-adrenal function. However, the simple and relatively crude test of adrenal responsiveness to tetracosactrin employed here appeared to provide a useful pointer to which patients were more likely to convert successfully. The nature of the physiological and/or psychological dependence on steroids which develops in RA patients whose symptoms are controlled by this means over long periods is unknown. Our results suggest that adrenal responsiveness is one significant factor. They also point to this as a useful screening test for deciding which patients should not be subjected to the discomfort of attempting conversion.

Is it worth attempting conversion? Our data provide no clear evidence of any reduction in the undesirable side effects of prednisolone as a result of conversion. However, the period of observation following establishment of the alternate daily regimen (about 12 weeks) was probably too short to show such effects. Such evidence as there is 245 suggests that the attempt is worth making. 


\section{References}

1 Harter J G, Reddy W J, Thorn G W. Studies on an intermittent corticosteroid dosage regimen. $N$ Engl $J$ Med 1963; 269: 591-6.

2 Carter M E, James V H T. Effect of alternate day, single dose corticosteroid therapy on pituitary adrenal function. Ann Rheum Dis 1972; 31: 379-83.

3 MacGregor R R, Sheagren J N, Lipsett M B, Wolff S M. Alternate day prednisone therapy: evaluation of delayed hypersensitivity responses, control of the disease and steroid side effects. N Engl J Med 1969; 280: 1427-31.
4 Dale D C, Fauci A S, Wolff S M. Alternate-day prednisone, leucocyte kinetics and susceptibility to infections. N Engl J Med 1974; 291 : 1154-8.

5 Fauci A S. Alternate-day corticosteroid therapy. Am J Med 1978; 64: 729-31.

6 Jasani M K, Boyle J A, Greig W R. Corticosteroidinduced suppression of the hypothalamo-pituitary-adrenal axis: observations on patients given oral corticosteroids for rheumatoid arthritis. $Q J$ Med 1967; 36: 261-76.

7 Ansell B, Bywaters E. Alternate-day corticosteroid therapy in juvenile chronic polyarthritis. $J$ Rheumatol 1974; 1 : 176-86. 